\title{
Treatment Complications and Long-term Outcomes of Total Body Irradiation in Patients with Acute Lymphoblastic Leukemia: A Single Institute Experience
}

\author{
FRANCESCA DE FELICE ${ }^{1}$, LAVINIA GRAPULIN ${ }^{1}$, DANIELA MUSIO ${ }^{1}$, JENNY POMPONI ${ }^{1}$, \\ CINZIA DI FELICE ${ }^{2}$, ANNA PAOLA IORI ${ }^{3}$, ALICE BERTAINA $^{4}$ and VINCENZO TOMBOLINI ${ }^{1,5}$ \\ Departments of ${ }^{1}$ Radiotherapy, and ${ }^{2}$ Medical Physics, and ${ }^{3}$ Department of Cellular Biotechnologies and Hematology, \\ Thrombosis Center, Policlinico Umberto I, Sapienza University of Rome, Rome, Italy; \\ ${ }^{4}$ Department of Pediatric Hematology and Oncology, IRCCS Bambino Gesù Children's Hospital, Rome, Italy; \\ ${ }^{5}$ Spencer-Lorillard Foundation, Rome, Italy
}

\begin{abstract}
Background: The aim of this study was to evaluate treatment-related toxicity and clinical outcomes of total body irradiation (TBI) in patients with acute lymphoblastic leukemia (ALL). Patients and Methods: We performed a retrospective review of all patients with ALL who underwent TBI-based conditioning regimen at our Institution between 2000 and 2012. Results: A total of 211 patients were included. The median follow-up was 40 months. The 5-year overall survival and disease-free survival were $64.7 \%$ and $62.8 \%$, respectively. The 5-year overall survival rate for the 163 children was $67.6 \% \quad(95 \%$ confidence interval $=55-77 \%)$. Disease status at time of transplant did not improve disease-free survival. Gastrointestinal acute toxicity was the most common early side-effect (19.9\%). Acute graft-versus-host disease was reported in 31 patients (14.7\%). Main late toxicities were cataract induction (12.8\%) and growth, gonadal and endocrine effects (36\%). Conclusion: TBI-based conditioning regimen led to a high survival rate with remarkably low radiation-related toxicity, suggesting that TBI provides a feasible therapeutic option in patients with ALL.
\end{abstract}

Acute lymphoblastic leukemia (ALL) is a relatively common hematological disease, with an estimated 770 new cases/year in Italy (1). ALL represents approximately $80 \%$ of leukemia cases among children and $20 \%$ among adults. In the past

Correspondence to: Francesca De Felice, Department of Radiotherapy, Policlinico Umberto I, Sapienza University of Rome, Viale Regina Elena 326, 00161 Rome, Italy. Tel: +39 0649973411, Fax: +390649973411, e-mail fradefelice@hotmail.it

Key Words: Total body irradiation, hematologic cancer, acute lymphoblastic leukemia, child, conditioning regimen, radiotherapy. several decades, survival outcomes have substantially improved, especially in children, due to advances in molecular genetics and treatment regimens $(1,2)$. Variability exists across studies with regard to the optimal conditioning regimen prior to hematopoietic stem cell transplantation (HSCT) (2).

Total body irradiation (TBI) is a specialized radiation therapy technique and, in conjunction with chemotherapy, has been found to be an effective and valid immunosuppressive and myeloablative treatment option for transplant preparation (3). TBI is advantageous because irradiation can be delivered uniformly and its radiobiological effects reach 'hermetic' sites, such as the central nervous system, without interference from metabolic processes and without chemotherapy cross-resistance (4). But there are worries concerning acute and late toxicity. TBI can improve oncological outcomes at the cost of intensified immediate side-effects and long-term complications of radiation (5).

At our institution, TBI has been routinely performed for years in conditioning regimen. The purpose of this study was to describe the long-term follow-up data of patients with ALL treated with a TBI conditioning regimen in order to analyze clinical outcomes, toxicity rates and prognostic factors.

\section{Patients and Methods}

Patient selection. Medical records of all consecutive patients who underwent TBI plus chemotherapy conditioning regimen for allogeneic HSCT between 2000 and 2012 at the Policlinico Umberto I Hospital, Sapienza University of Rome, were retrospectively reviewed. The year 2012 was chosen to allow adequate follow-up. The conditioning regimen was reviewed and approved by the Institutional Review Board and the Scientific Review Committee (Prot. 306/16). Written informed consent was obtained from all patients and, in the case of a child, the patient's parents before initiation of therapy. 
In total, 280 patients presented with various hematological malignancies. Because of specific histology and different natural history, chronic lymphoblastic leukemia $(n=7)$, myelogenous leukemia $(n=29)$, non-Hodgkin lymphoma $(n=12)$, multiple myeloma $(n=10)$ and other undifferentiated leukemia types $(n=11)$ were excluded. Overall, the final analysis was restricted to those 211 patients with ALL who received TBI plus chemotherapy conditioning regimen for allogeneic HSCT. The diagnosis of ALL was confirmed by histological and immunophenotypic review of the initial diagnostic bone marrow aspirate specimen.

TBI procedure was performed at our Department, whereas chemotherapy regimens and HSCT were performed either at our Department or at our affiliated hospital, Bambin Gesù of Rome.

Data collected included demographics, initial diagnosis and disease status at time of HSCT, treatment modalities and follow-up. According to the National Cancer Institute, patients older than 15 years were considered young adults/adults (6). Information regarding TBI regimen, including dose, fractionation, and toxicity, were also recorded.

Conditioning regimen. Conditioning regimen consisted of sequential administration of chemotherapy and TBI.

Chemotherapy was started 21 days before HSCT. The chemotherapy regimen was left at the oncologist's discretion. Heterogeneous chemotherapy regimens were employed and comprised either a single agent or a combination of agents, including cyclophosphamide, etoposide and thiotepa, principally.

Fractionated TBI was completed 4 days before HSCT and was delivered over 3 consecutive days. Patients received a total dose of $12 \mathrm{~Gy}$, given in six fractions within 3 days, two fractions/day with 6 hours minimum interval between fractions. Treatment simulation was made with total body computed tomography (CT) and $1 \mathrm{~cm}$ thickness slices. During data acquisition and during treatment, the patient lay in a supine and lateral decubitus position, with arms across the chest. To calculate the dose distribution and to define the dose prescription point, measurements of body thickness were taken at the upper and lower lung, abdomen, pelvis and ankle. The patient was positioned at an extended distance from the linear accelerator's beam to cover the whole body. Perspex shields were placed at the edge of the treatment couch between patient and linear accelerator to homogenize the dose, according to the different thicknesses of the body and reduce the dose to the lung. Antero-posterior and lateral-lateral beams were used.

Before each TBI fraction, patients received a prophylactic therapy, based on desamethasone and ondansetron, to reduce and prevent radiation toxicity.

Graft-versus-host disease (GVHD) prophylaxis consisted of cyclosporine with or without methotrexate with/without prednisone with/without antithymocyte globulin.

Toxicity. Treatment-related toxicity was evaluated according to Common Terminology Criteria for Adverse Events, Version 4.0 (CTCAE v4) (7). GVHD was graded using the Consensus Conference on Acute GVHD Grading criteria, depending on the degree of organ involvement (8).

Statistical analysis. Statistical analysis was carried out using RStudio 0.98.1091 software. Standard descriptive statistics were used to evaluate the distribution of each factor. Continuous variables are presented as means and ranges, and dichotomous variables are presented as percentages. Quantitative variables were compared using Student $t$-test, whereas percentages were compared using the chi-square test.

Overall survival (OS) and disease-free survival (DFS) were calculated in months from the date of the end of TBI to the first event, including date of the last follow-up or death (OS), or persistent or relapsed disease after transplant (DFS).

OS and DFS were estimated using the Kaplan-Meier method and survival curves were compared using the log-rank statistic test. Potential confounding factors included difference in chemotherapy regimens and in follow-up times, and they were controlled by using a random-effects model.

The following variables were investigated: age in years at conditioning regimen before transplantation $(\leq 15 v s .>15)$, sex (male vs. female), disease status at time of HSCT (first complete remission $v s$. second $v s$. higher remission), type of conditioning regimen (cyclophosphamide-TBI vs. thiotepa-TBI). Variables associated with a $p$-value of 0.25 or less were included in a multivariate survival Cox regression analysis. All reported $p$-values are two-sided, and those lower than 0.05 were considered significant.

\section{Results}

Patient characteristics. Two hundred and eleven patients were eligible for this study. Patient baseline characteristics and primary disease distribution are shown in Table I. Median age at diagnosis was 14 years (range=3-53) and 145 patients $(68.7 \%)$ were male. The vast majority of patients $(\mathrm{n}=89 ; 42.2 \%)$ received HSCT in second complete remission by pre-transplantation conditioning regimen. In total, 163 patients $(77.3 \%)$ were 15 years old or less, while 48 patients $(22.7 \%)$ were young adults/adults. There were no significant differences between children and young adults/adults in term of sex and disease status at time of HSCT.

Compliance. Compliance with treatment was excellent. No TBI breaks were recorded. A total of 117 patients $(55.4 \%)$ received cyclophosphamide-based conditioning chemotherapy, whereas in the remainder, chemotherapy was based on thiotepa (85 cases; $40.3 \%$ ) or other drugs (nine cases; $4.3 \%$ ).

Toxicity. Toxicity is listed in Table II. Gastrointestinal acute toxicity, including nausea, vomiting and diarrhea were the most common early side-effects $(n=42 ; 19.9 \%)$. It was evaluated as moderate (grade $\leq 2$ ), but in was grade 3 in one patient. Cutaneous $(n=18)$, liver $(n=5)$ and gastrointestinal $(n=8)$ acute GVHD was reported in a total of 31 patients $(14.7 \%)$, with grade $1-2,3$ and 2 , respectively. It occurred more frequently in children than young adults/adults $(19 \%$ vs. $0 \%, p=0.01)$.

Main late toxicities were cataract induction $(n=27$; $12.8 \%)$, and growth, gonadal and endocrine effects $(n=76$; $36 \%$ ). Ocular complications were observed at mean time of 40 months (range=17-97 months), whereas endocrine complications occurred at a mean time of 25 months (range=9-89 months). Renal failures were not recorded. No 
Table I. Baseline patient characteristics.

\begin{tabular}{|c|c|c|c|c|}
\hline \multirow[b]{2}{*}{ Characteristic } & \multicolumn{3}{|c|}{ Patient, n (\%) } & \multirow[b]{2}{*}{$p$-Value } \\
\hline & Total & Children & Young adults/adults & \\
\hline Gender & & & & 0.22 \\
\hline Male & $145(68.7)$ & $112(68.7)$ & $33(68.8)$ & \\
\hline Female & $66(31.3)$ & $51(31.3)$ & $15(31.2)$ & \\
\hline Median age (range), years & $14(3-53)$ & $9(3-15)$ & $25(16-53)$ & \\
\hline ALL & $211(100)$ & $163(100)$ & $48(100)$ & \\
\hline Disease status at HSCT & & & & 0.35 \\
\hline First complete remission & $66(31.3)$ & $45(27.6)$ & $21(43.8)$ & \\
\hline Second complete remission & $89(42.2)$ & $77(47.2)$ & $12(25)$ & \\
\hline Third or more complete remission & $37(17.5)$ & $28(17.2)$ & $9(18.7)$ & \\
\hline Persistent molecular disease & $19(9)$ & $13(8)$ & $6(12.5)$ & \\
\hline
\end{tabular}

ALL: Acute lymphoid leukemia; HSTC: hematopoietic stem cell transplantation.

Table II. Acute and delayed toxicity after total body irradiation for preconditioning in patients with acute lymphoid leukemia.

\begin{tabular}{|c|c|c|c|c|}
\hline \multirow[b]{2}{*}{ Toxicity } & \multicolumn{3}{|c|}{ Patients, n (\%) } & \multirow[b]{2}{*}{$p$-Value } \\
\hline & Total & Children & Young adults/adults & \\
\hline \multicolumn{5}{|l|}{ Constitutional symptom } \\
\hline Fatigue & $6(2.8)$ & $4(2.5)$ & $2(4.2)$ & 0.44 \\
\hline \multicolumn{5}{|l|}{ Dermatology/skin } \\
\hline Radiation dermatitis & $3(1.4)$ & $2(1.2)$ & $1(2.1)$ & 0.99 \\
\hline \multicolumn{5}{|l|}{ Gastointestinal } \\
\hline Diarrohea & $19(9)$ & $16(9.8)$ & $3(6.3)$ & 0.52 \\
\hline Nausea and vomiting & $23(10.9)$ & $14(8.5)$ & $9(18.8)$ & 0.09 \\
\hline Mouth dryness & $7(3.3)$ & $4(2.5)$ & $3(6.3)$ & 0.14 \\
\hline Mucositis & $8(3.8)$ & $8(4.9)$ & $0(0)$ & 0.22 \\
\hline Parotitis & $12(5.7)$ & $7(4.3)$ & $5(10.4)$ & 0.48 \\
\hline \multicolumn{5}{|l|}{ Endocrine } \\
\hline Hypothyroidism & $24(11.4)$ & $20(12.3)$ & $4(8.3)$ & 0.33 \\
\hline Growth dysfunction & $25(11.8)$ & $25(15.3)$ & $0(0)$ & 0.10 \\
\hline Sterility/infertility & $27(12.8)$ & $22(13.5)$ & $5(10.4)$ & 0.11 \\
\hline \multicolumn{5}{|l|}{ Hepatic } \\
\hline VOD/SOS & $35(16.6)$ & $27(16.5)$ & $8(16.7)$ & 0.24 \\
\hline \multicolumn{5}{|l|}{ Ocular/visual } \\
\hline Cataract & $27(12.8)$ & $22(13.5)$ & $5(10.4)$ & 0.71 \\
\hline \multicolumn{5}{|l|}{ Pulmunary } \\
\hline Interstitial pneumonitis & $19(9)$ & $15(7.2)$ & $4(8.3)$ & 0.88 \\
\hline Secondary malignancy & $0(0)$ & $0(0)$ & $0(0)$ & \\
\hline GVHD & & & & 0.01 \\
\hline Acute & $31(14.7)$ & $31(19)$ & $0(0)$ & \\
\hline Chronic & $10(4.7)$ & $10(6.1)$ & $0(0)$ & \\
\hline
\end{tabular}

VOD/SOS: Veno-occlusive disease/sinusoidal obstructive syndrome; GVHD: graft-versus-host disease.

evidence of a second tumor occurred. Chronic GVHD developed in $4.7 \%$ of patients $(n=10)$, whereas engraftment failure was seen in $10.4 \%$ of cases $(n=22)$. These two complications were related to an increased risk of lung toxicity ( $28 \% v s .13 \% ; p=0.03)$.
OS and prognostic factors. Overall 58 patients (27.5\%) had died by study end. After a median follow-up of 40 months, the 5 -year OS was estimated at $64.7 \%$ (95\% confidence interval $(\mathrm{CI})=56-73 \%$ ] (Figure 1). The prognostic analysis for OS is shown in Table III. In the multivariate analysis, there were no 


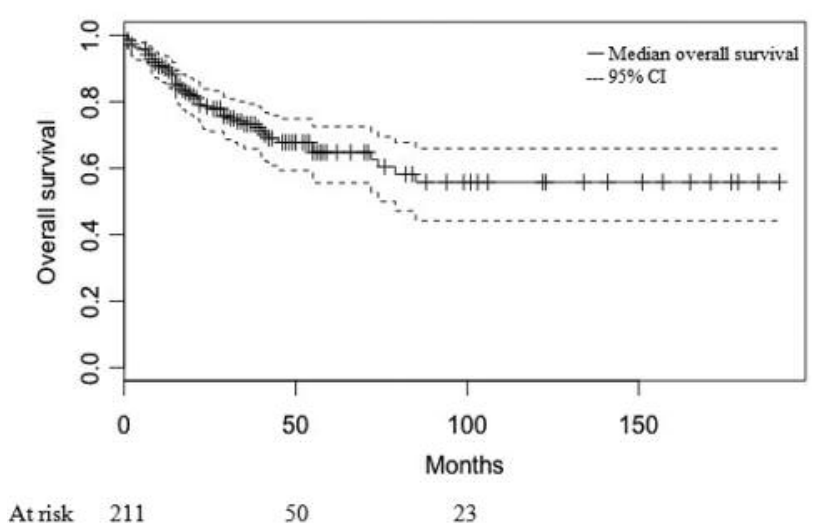

Figure 1. Overall survival for the whole cohort.

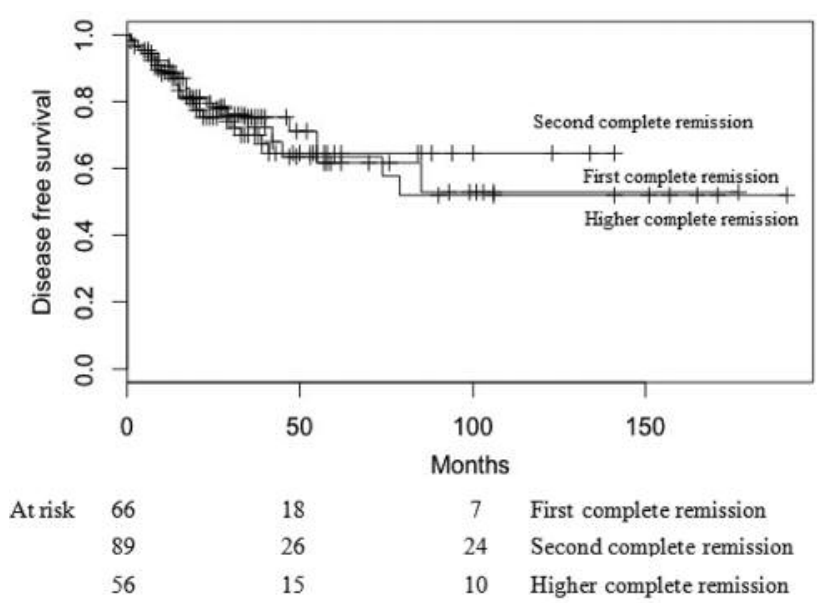

Figure 2. Disease-free survival according to disease status at time of hematopoietic stem cell transplantation.

predictors of OS. The major, but not significant, prognostic factor was age, with a 5-year OS of $67.6 \%$ (95\% CI=55-77\%) and $58.5 \%$ (95\% CI=44-71\%) for children and young adults/adults, respectively $(p=0.25)$. No significant differences in OS were found regarding sex, type of chemotherapy and disease status at time of HSCT, even after adjustment by age.

DFS and causes of death. The 5-year DFS rate was 62.8\% (95\% CI $=53-71 \%)$. Overall the disease status at time of HSCT was not strongly associated with DFS $(p>0.05)$, as shown in Figure 2. Of the 58 deaths by study end, 43 (74.1\%) were relapse-related. Other causes of death were related to transplant-related mortality $(\mathrm{n}=15 ; 25.9 \%)$, including pneumonia $(n=4)$, graft failure $(n=5)$, fungal infection $(n=4)$ and acute respiratory distress syndrome $(n=2)$. There were no differences regarding age, sex and type of chemotherapy.

\section{Discussion}

Our local policy is to perform TBI-based conditioning regimen as part of curative treatment, considering the high radiosensitivity of ALL. We reported on a large cohort of patients with ALL treated with TBI conditioning regimen in the last 12 years at our Institution. Irradiation ensured a relative good survival rates within tolerable toxicity, independently of disease status at the time of HSCT and type of chemotherapy regimen associated. Age of 15 years or more was favorably associated with superior 5-year OS (67.6\% vs. $58.5 \%, p=0.25)$. This positive trend was consistent with the outcomes reported in literature. Several studies recently published have emphasized the importance of age in OS $(9,10)$. For example, Mikell et al. reported on a series of 83 patients with a diagnosis of ALL who underwent TBI as conditioning for HSCT. In their series, lower age was associated with improved OS (hazard ratio $=1.04,95 \% \mathrm{CI}=1.01-1.08, p=0.025)$ (9). Similarly, Marks et al. reported on a series of 298 patients with ALL in first or second complete remission after pre transplantation conditioning regimen with chemo-TBI. They showed that age $>20$ years was associated with increased mortality (hazard ratio $=1.69,95 \% \mathrm{CI}=1.27-2.25, p=0.0003$ ) (10).

Although it appears that disease status at transplant is useful in predicting poor prognosis, with advanced stage, including second, third or fourth complete remission and refractory or relapsed disease being related to lower OS and DFS $(11,12)$, in our series there were no significant survival benefits even after adjustment for the complete remission status. These differences in the observed versus expected survival rates may be secondary to the fact that a large proportion of patients with ALL in our cohort were in second or higher complete remission, which has been identified as a high-risk treatment group.

Analysis of toxicity indicators reflected that the TBI regimen was generally well tolerated, with high compliance rate and good level of toxicity. Severe acute toxicities were negligible. The overall low rate of long-term complications observed in our study was the main end result. This might be related to the routine use of appropriate devices such as lung shielding to homogenize the dose.

To reduce typical TBI dose inhomogeneities, especially for the lung, intensity-modulated radiotherapy (IMRT) TBI has been proposed (13). Schneider et al. treated a total of 257 patients with different hematologicaI malignancies with IMRT TBI. After a median follow-up of 9.2 years, the main late toxicities were interstitial pneumonitis (10.9\%), veno-occlusive disease/sinusoidal obstructive syndrome (VOD/SOS) (5.8\%), nephrotoxicity (3.5\%), cataract (6.6\%), chronic GVHD $(17.8 \%)$, thyroid gland disorders $(3.1 \%)$, growth retardation 
Table III. Univariate and multivariate analyses of prognostic factors for overall survival.

\begin{tabular}{|c|c|c|c|c|}
\hline \multirow[b]{2}{*}{ Factor } & \multicolumn{2}{|c|}{ Univariate analysis } & \multicolumn{2}{|c|}{ Multivariate analysis } \\
\hline & $\mathrm{HR}(95 \% \mathrm{CI})$ & $p$-Value & HR $(95 \%$ CI $)$ & $p$-Value \\
\hline Gender (male $v s$. female) & $0.68(0.30-7.05)$ & 0.63 & & \\
\hline Age $(<15 v s . \geq 15$ years $)$ & $0.81(0.53-1.22)$ & 0.25 & $0.79(0.52-1.20)$ & 0.65 \\
\hline Disease status (first CR vs. second vs. higher) & $0.75(0.76-2.31)$ & 0.21 & $0.72(0.73-2.29)$ & 0.32 \\
\hline CHT regimen (Cy-TBI vs. thiotepa-TBI) & $0.76(0.34-4.95)$ & 0.69 & & \\
\hline
\end{tabular}

HR: Hazard ratio; CI: confidence interval; CR: complete remission; CHT: chemotherapy; Cy: cyclophosphamide; TBI: total body irradiation.

(12.5\%) and secondary malignancy (4.3\%) (13). The German experience did not show a significant reduction of late toxicities and data were in agreement with our results, but for second malignancy. In fact, considering that the latency of radiation-induced malignancy is more than 10 years, probably a longer follow-up is necessary in order to correctly correct evaluate risk in our cohort (14).

Generally, the major criticism of TBI-based conditioning regimen is the radiation late sequelae. Survivors of TBIconditioning regimen may develop a number of complications linked to organ failure, such as hypogonadism, growth hormone deficiency and hypothyroidism, likely because radiation affects cells differentiation and metabolic status (15). During recent years, several clinical trials have compared TBI-conditioning regimen to high-dose chemotherapy-based regimens for HSCT (16-20). Data were collected and summarized in a published meta-analysis (21). Overall, 652 patients were included in the meta-analysis, of whom 314 received TBI and 338 received high-dose chemotherapy. Results showed that TBI-based regimens were superior for both OS and DFS, but the advantages over high-dose chemotherapy regimens were not statistically significant (odds ratio $(\mathrm{OR})=1.4,95 \% \mathrm{CI}=0.9-2.2$ for $\mathrm{OS}$; $\mathrm{OR}=1.2,95 \% \mathrm{CI}=0.7-2.1$ for DFS). Toxicities, including GVHD and interstitial pneumonitis, did not differ significantly, whereas there was a significantly higher incidence of $\mathrm{VOD} / \mathrm{SOS}$ in patients who underwent chemotherapy regimens $(\mathrm{OR}=2.5,95 \% \mathrm{CI}=1.2-5.2, p=0.02)$. Thus, the authors concluded that TBI could be used in conditioning regimens. The limitations of the analysis were mostly related to the heterogeneity of trials, regarding patient population, treatments and follow-up period. However, the lack of actual data on late effects of high-dose chemotherapy limits definitive conclusions.

Considering the significant homogeneity of TBI regimens used prior to HSCT at our Institution, we support the role of TBI in maximizing disease control and reducing the potential treatment-related toxicity. The retrospective nature of the study introduced some limitation in the interpretation of long-term survival and toxicity data. In order to control hematological disease heterogeneity and thus reduce patient selection bias, we generated a homogeneous cohort of all patients with ALL. We lacked prospectively acquired basal clinical features which limited our ability to perform a more detailed prognostic factors analysis. On the other hand, our cohort can boast uniformity of the TBI-based conditioning regimen and its prophylaxis.

However, the question of how a TBI-based conditioning regimen would compare with chemotherapy-based regimes remains, especially in long-term follow-up data. In terms of late toxicity, the published experience of chemotherapybased regimens for ALL is not as extensive or mature as it is for TBI-based regimen. Our analysis demonstrated the lack of severe acute and late toxicity, suggesting that TBI may be a potential safe therapeutic option. This study could be used as contribution for designing innovative treatment regimens for patients with ALL patients in the near future.

\section{Conclusion}

TBI provides an effective conditioning regimen for HSCT. Our study confirmed high survival rates, without severe acute and late toxicity, for patients with ALL treated with a TBI-based conditioning regimen.

\section{References}

1 Linee guida Leucemia Linfoblastica Acuta Associazione Italiana Ematologia Oncologica Pediatrica 2015. [http://www.aieop.org/]

2 National Comprehensive Cancer Network Guidelines Acute Lymphoblastic Leukemia Version 2.2015. [http://www.nccn.org/]

3 Davies SM, Ramsay NK, Klein JP, Weisdorf DJ, Bolwell B, Cahn JY, Camitta BM, Gale RP, Giralt S, Heilmann C, HensleeDowney PJ, Herzig RH, Hutchinson R, Keating A, Lazarus HM, Milone GA, Neudorf S, Perez WS, Powles RL, Prentice HG, Schiller G, Socié G, Vowels M, Wiley J, Yeager A and Horowitz MM: Comparison of preparative regimens in transplants for children with acute lymphoblastic leukemia. J Clin Oncol 18(2): 340-347, 2000 . 
4 Copelan EA and Deeg HJ: Conditioning for allogeneic marrow transplantation in patients with lymphohematopoietic malignancies without the use of total body irradiation. Blood 80(7): 1648-1658, 1992.

5 Eroglu C, Pala C, Kaynar L, Yaray K, Aksozen MT, Bankir M, Zararsız G, Orhan O, Gündog M, Yıldız OG, Eser B, Cetin M and Unal A: Comparison of total body irradiation plus cyclophosphamide with busulfan plus cyclophosphamide as conditioning regimens in patients with acute lymphoblastic leukemia undergoing allogeneic hematopoietic stem cell transplant. Leuk Lymphoma 54(11): 2474-2479, 2013.

6 Geiger AM and Castellino SM: Delineating the age ranges used to define adolescents and young adults. J Clin Oncol 29(16): e492-3, 2011.

7 Cancer Therapy Evaluation Program (2010) Common Terminology Criteria for Adverse Events, Version 4.0. http://ctep.cancer.gov.

8 Przepiorka D, Weisdorf D, Martin P, Klingemann HG, Beatty P, Hows J and Thomas ED: 1994 Consensus Conference on Acute GVHD Grading. Bone Marrow Transplant 15(6): 825-828, 1995.

9 Mikell JL, Waller EK, Switchenko JM, Rangaraju S, Ali Z, Graiser M, Hall WA, Langston AA, Esiashvili N, Khoury HJ and Khan MK: Similar survival for patients undergoing reducedintensity total body irradiation (TBI) versus myeloablative TBI as conditioning for allogeneic transplant in acute leukemia. Int J Radiat Oncol Biol Phys 89(2): 360-369, 2014.

10 Marks DI, Forman SJ, Blume KG, Pérez WS, Weisdorf DJ, Keating A, Gale RP, Cairo MS, Copelan EA, Horan JT, Lazarus HM, Litzow MR, McCarthy PL, Schultz KR, Smith DD, Trigg ME, Zhang MJ and Horowitz MM: A comparison of cyclophosphamide and total body irradiation with etoposide and total body irradiation as conditioning regimens for patients undergoing sibling allografting for acute lymphoblastic leukemia in first or second complete remission. Biol Blood Marrow Transplant 12(4): 438-453, 2006.

11 Mori T, Aisa Y, Kato J, Yamane A, Nakazato T, Shigematsu N and Okamoto S: Safety and efficacy of total body irradiation, cyclophosphamide, and cytarabine as a conditioning regimen for allogeneic hematopoietic stem cell transplantation in patients with acute lymphoblastic leukemia. Am J Hematol 87(4): 349$353,2012$.

12 Ramanujachar R, Richards S, Hann I, Goldstone A, Mitchell C, Vora A, Rowe J and Webb D: Adolescents with acute lymphoblastic leukaemia: outcome on UK national paediatric (ALL97) and adult (UKALLXII/E2993) trials. Pediatr Blood Cancer 48(3): 254-261, 2007.

13 Schneider RA1, Schultze J, Jensen JM, Hebbinghaus D and Galalae RM: Long-term outcome after static intensity-modulated total body radiotherapy using compensators stratified by pediatric and adult cohorts. Int J Radiat Oncol Biol Phys 70(1): 194-202, 2008.
14 Tucker MA, Meadows AT, Boice JD Jr, Stovall M, Oberlin O, Stone BJ, Birch J, Voûte PA, Hoover RN and Fraumeni JF Jr.: Leukemia after therapy with alkylating agents for childhood cancer. J Natl Cancer Inst 78(3): 459-464, 1987.

15 Rajendran R, Abu E, Fadl A and Byrne CD: Late effects of childhood cancer treatment: severe hypertriglyceridaemia, central obesity, non alcoholic fatty liver disease and diabetes as complications of childhood total body irradiation. Diabet Med 30(8): e239-242, 2013.

16 Blaise D, Maraninchi D, Archimbaud E, Reiffers J, Devergie A, Jouet JP, Milpied N, Attal M, Michallet M and Ifrah N: Allogeneic bone marrow transplantation for acute myeloid leukemia in first remission: a randomized trial of a busulfanCytoxan versus Cytoxan-total body irradiation as preparative regimen: a report from the Group d'Etudes de la Greffe de Moelle Osseuse. Blood 79(10): 2578-2582, 1992.

17 Clift RA, Buckner CD, Thomas ED, Bensinger WI, Bowden R, Bryant E, Deeg HJ, Doney KC, Fisher LD and Hansen JA: Marrow transplantation for chronic myeloid leukemia: a randomized study comparing cyclophosphamide and total body irradiation with busulfan and cyclophosphamide. Blood 84(6): 2036-2043, 1994.

18 Devergie A, Blaise D, Attal M, Tigaud JD, Jouet JP, Vernant JP, Bordigoni P, Ifrah N, Dauriac C and Cahn JY: Allogeneic bone marrow transplantation for chronic myeloid leukemia in first chronic phase: a randomized trial of busulfan-cytoxan versus cytoxan-total body irradiation as preparative regimen: a report from the French Society of Bone Marrow Graft (SFGM). Blood 85(8): 2263-2268, 1995.

19 Ringdén O, Ruutu T, Remberger M, Nikoskelainen J, Volin L, Vindeløv L, Parkkali T, Lenhoff S, Sallerfors B and Ljungman P: A randomized trial comparing busulfan with total body irradiation as conditioning in allogeneic marrow transplant recipients with leukemia: a report from the Nordic Bone Marrow Transplantation Group. Blood 83(9): 2723-2730, 1994.

20 Blume KG, Kopecky KJ, Henslee-Downey JP, Forman SJ, Stiff PJ, LeMaistre CF and Appelbaum FR: A prospective randomized comparison of total body irradiation-etoposide versus busulfancyclophosphamide as preparatory regimens for bone marrow transplantation in patients with leukemia who were not in first remission: a Southwest Oncology Group study. Blood 81(8): 2187-2193, 1993.

21 Hartman AR, Williams SF and Dillon JJ: Survival, disease-free survival and adverse effects of conditioning for allogeneic bone marrow transplantation with busulfan/cyclophosphamide vs total body irradiation: a meta-analysis. Bone Marrow Transplant 22(5): 439-443, 1998.

Received July 16, 2016

Revised August 1, 2016

Accepted August 4, 2016 